\title{
Large spinal intraosseous arteriovenous fistula: case report
}

\author{
Yasuaki Imajo, MD, Tsukasa Kanchiku, MD, Yuichiro Yoshida, MD, Norihiro Nishida, MD, \\ and Toshihiko Taguchi, MD
}

Department of Orthopaedic Surgery, Yamaguchi University Graduate School of Medicine, Yamaguchi, Japan

\begin{abstract}
Here the authors report the case of a fresh vertebral body fracture with a large spinal intraosseous arteriovenous fistula (AVF). A 74-year-old woman started to experience low-back pain following a rear-end car collision. Plain radiography showed diffuse idiopathic skeletal hyperostosis (DISH). Sagittal CT sections revealed a transverse fracture of the L-4 vertebral body with a bone defect. Sagittal fat-suppressed T2-weighted MRI revealed a flow void in the fractured vertebra. Spinal angiography revealed an intraosseous AVF with a feeder from the right L-4 segmental artery. A fresh fracture of the L-4 vertebral body with a spinal intraosseous AVF was diagnosed. Observation of a flow void in the vertebral body on fat-suppressed T2-weighted MRI was important for the diagnosis of the spinal intraosseous AVF. Because conservative treatment was ineffective, surgery was undertaken. The day before surgery, embolization through the right L-4 segmental artery was performed using 2 coils to achieve AVF closure. Posterolateral fusion with instrumentation at the T12-S2 vertebral levels was performed without L-4 vertebroplasty. The spinal intraosseous AVF had disappeared after 4 months. At 24 months after surgery, the bone defect was completely replaced by bone and the patient experienced no limitations in daily activities. Given their experience with the present case, the authors believe that performing vertebroplasty or anterior reconstruction may not be necessary in treating spinal intraosseous AVF.
\end{abstract}

http://thejns.org/doi/abs/10.3171/2014.10.SPINE1487

KEY WORDS spinal intraosseous arteriovenous fistula; surgical treatment; lumbar spine; oncology

$\mathrm{T}$ HE occurrence of epidural or paraspinal arteriovenous fistula (AVF) is rare, ${ }^{3}$ and that of spinal intraosseous AVF is extremely rare. Only 2 cases of spinal intraosseous AVF associated with a fracture of the vertebral body have been reported in the English literature. ${ }^{1,2}$ One of the 2 cases $^{1}$ had a fracture of the L-3 vertebral body with a large flow void. In general, spinal reconstruction is required in such cases. However, the details of spinal reconstruction, functional recovery, and remodeling of the L-3 vertebra after endovascular surgery were not reported for this case. In addition, there are no reports related to fresh fractures associated with spinal intraosseous AVF in patients with diffuse idiopathic skeletal hyperostosis (DISH) patients. Although spinal reconstruction and embolization are important for treating vertebral AVF, spinal reconstruction and vertebral remodeling after endovascular surgery without spinal reconstruction were not discussed in the 2 previously published cases. Here, we describe the treatment of a large spinal intraosseous AVF associated with the fracture of the L-4 vertebral body. Informed consent was obtained from the patient. This case report was approved by the institutional review board of Yamaguchi University Hospital.

\section{Case Report}

History

A 74-year-old woman started experiencing low-back pain following a rear-end car collision. She was in the driver's seat at the time of the collision and was wearing a seatbelt. Her primary complaint was low-back pain. Upon admission, she showed tenderness at the lower back and could not walk because of low-back pain. Her neurological examination was normal without any motor or sensory deficits in the lower extremities. She was initially treated conservatively. After 3 weeks of bed rest, she was mobilized with a lumbar orthosis. However, the severe low-back pain persisted, and union of the L-4 vertebral body was not achieved 3 months after trauma; hence, she was referred to our hospital for surgery.

\section{Radiological Findings}

Plain radiographs in the anteroposterior view showed that the L-5 transverse processes had fused to the iliac crest bilaterally. In the lateral view, separation of the anterior part of the L-4 vertebral body was observed. Sagittal CT sections revealed a transverse fracture of the ver-

ABBREVIATIONS AVF = arteriovenous fistula; DISH = diffuse idiopathic skeletal hyperostosis. SUBMITTED January 24, 2014. ACCEPTED October 6, 2014.

INCLUDE WHEN CITING Published online January 30, 2015; DOI: 10.3171/2014.10.SPINE1487.

DISCLOSURE The authors report no conflict of interest concerning the materials or methods used in this study or the findings specified in this paper. 
tebral body with a bone defect in the L-4 vertebral body (Fig. 1). Bone union was visible from T11-12 to the L3-4 facet joint, suggesting that the patient had DISH. Sagittal fat-suppressed T2-weighted MRI revealed a flow void in the L-4 vertebral body (Fig. 2). Contrast-enhanced CT showed a large cavity and strong signal occurring immediately after aortic enhancement (Fig. 3). Spinal angiography revealed an intraosseous AVF with a feeder from the right L-4 segmental artery. The contrast agent within the cavity appeared to be draining into the epidural venous plexus.

A fresh fracture of the L-4 vertebral body with a spinal intraosseous AVF was diagnosed; therefore, we decided to perform surgery. The day before surgery, embolization through the right L-4 segmental artery was performed using 5- and 4-mm interlock detachable coils (Tornado Embolization Microcoil) to achieve AVF closure. Final angiography confirmed total obliteration of the AVF.

\section{Operation}

We performed posterolateral fusion with instrumentation at the T12-S2 levels using a navigation system (Medtronic Inc.), although we did not perform an L-4 vertebroplasty. The instrumentation was made from titanium alloys. Bone was harvested from the iliac crest and the L1-L5 spinous processes and was transferred to the transverse processes and the laminae of L-3, L-4, and L-5. The total operation time was 7 hours 29 minutes, and intraoperative blood loss was $1500 \mathrm{ml}$.

\section{Postoperative Course}

No neurological deficit was observed after surgery, and

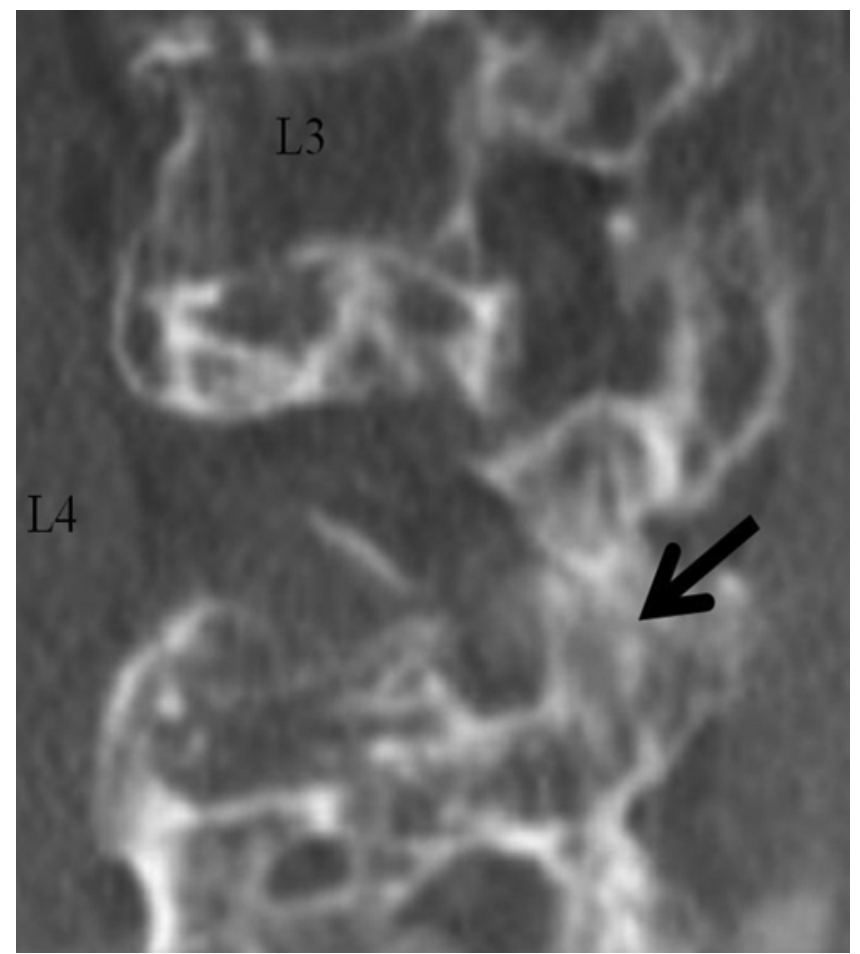

FIG. 1. Sagittal CT showed a fracture (arrow) and a large bone defect in the L-4 vertebral body.

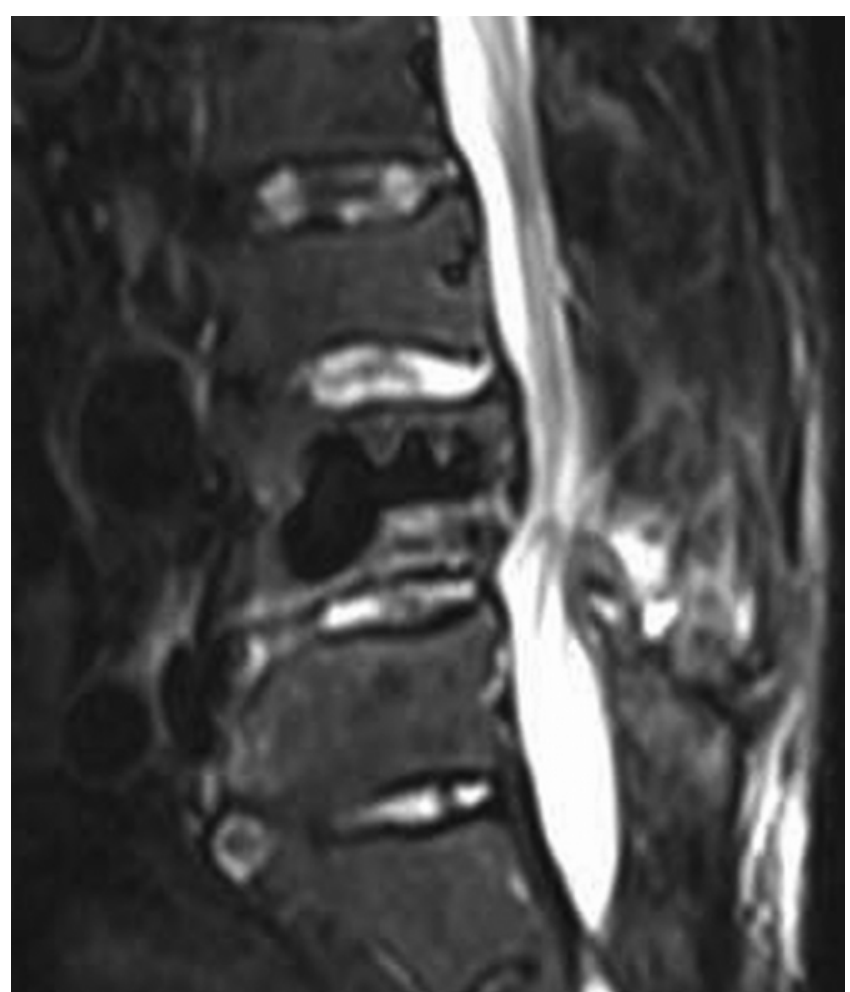

FIG. 2. Sagittal fat-suppressed T2-weighted MR image showed a flow void in the L-4 vertebral body and a high intensity area in the inferior of the L-4 vertebra. A fresh fracture was also noted in the L-4 vertebra.

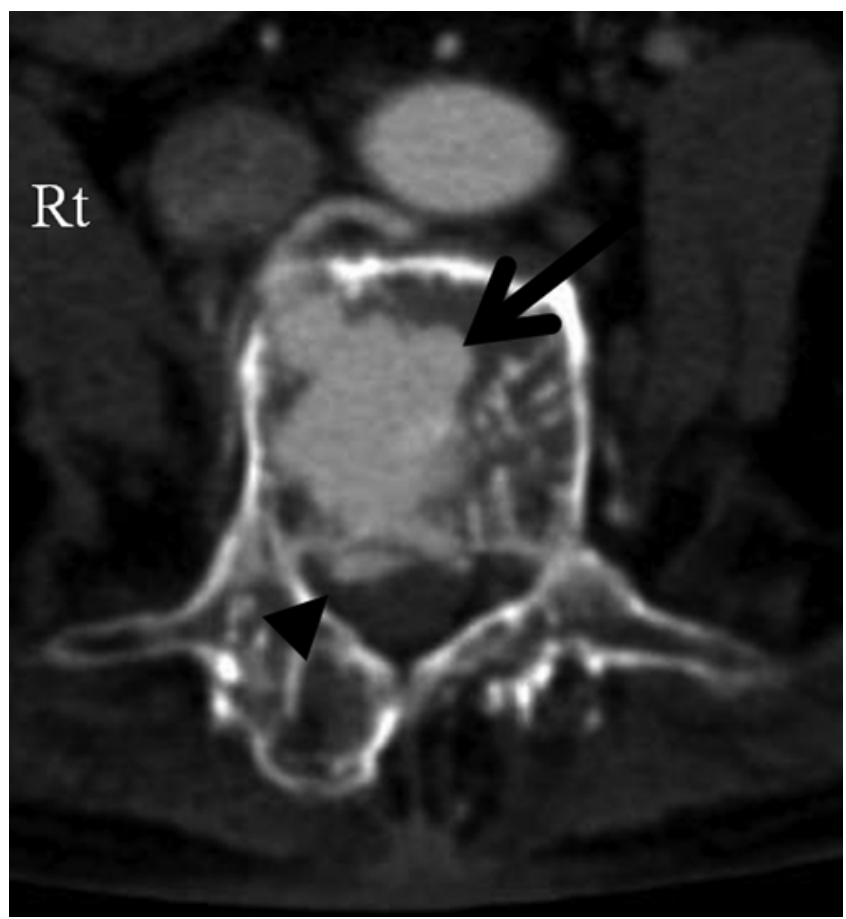

FIG. 3. Contrast-enhanced CT showed a hyper-enhanced cavity (arrow) in the L-4 vertebral body with inflow from the right L-4 segmental artery to a hyper-enhanced cavity and the epidural venous plexus (arrowhead). 


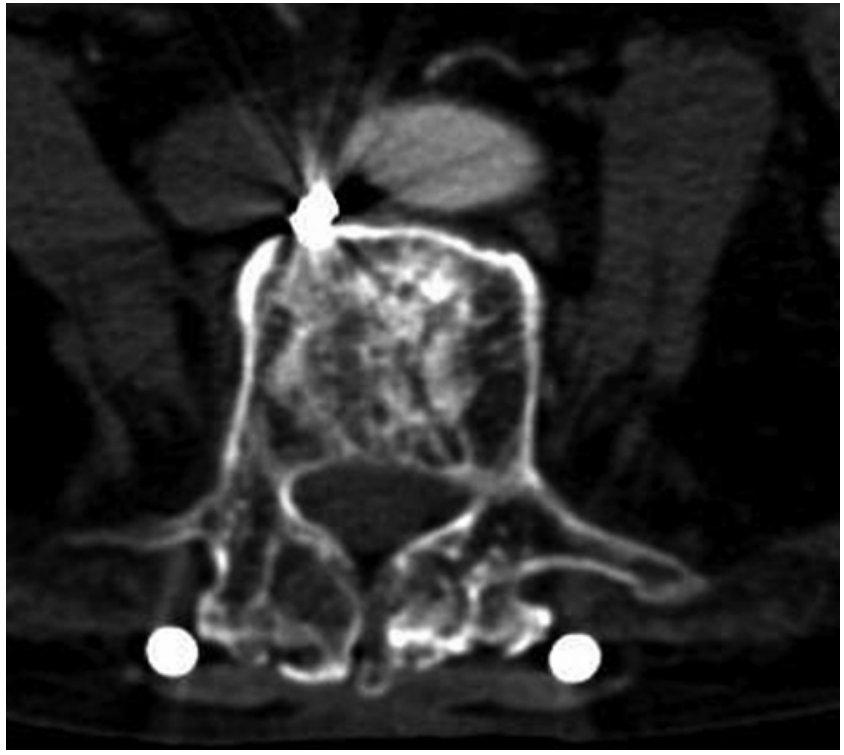

FIG. 4. Contrast-enhanced CT showed that the hyper-enhanced cavity in the L-4 vertebral body had disappeared 24 months postoperatively and was replaced by bone.

the patient was able to walk unassisted 2 months after surgery. Contrast-enhanced CT 3 weeks after surgery showed that the flow void in the L-4 vertebra had decreased. This void disappeared after 4 months, and part of the bone defect had been replaced by bone after 8 months. After 24 months, the defect was completely replaced by bone (Fig. 4). The patient experienced no restriction of daily activities 24 months after surgery.

\section{Discussion}

Because the flow void immediately after the car accident was very large on MRI and CT, we believed that the spinal intraosseous AVF of the L-4 vertebra was present before the accident. Because the patient had union at all levels between the T-11 vertebra and the pelvis, concentration of the stress could have caused the fracture of the L-4 vertebra. Because the patient had spinal intraosseous AVF of the L-4 vertebra before the car accident, we believed that the L-4 vertebra was biomechanically weak. Spinal intraosseous AVF was not diagnosed on the basis of radiographic and CT findings alone; the flow void and a fresh fracture on fat-suppressed T2-weighted MRI were essential for definitive diagnosis. Heavy intraoperative bleeding may have occurred if we had not performed prior embolization. We did not perform vertebroplasty because there were cortical bone defects on the anterior and posterior sides of the L-4 vertebral body. Papadoulas et al. reported on vascular injury complicating lumbar disc surgery. ${ }^{4} \mathrm{Ar}-$ teriovenous fistula was diagnosed more than 1 month after surgery in 66 patients, most of whom clinically presented with congestive heart failure. The mortality rate in their report was $5 \%$. Risk factors were improper positioning of the patient and vertebral anomalies such as hypertrophic spurs. Endovascular techniques are safe and effective for the treatment of AVF. In the present case, intraoperative spinal intraosseous AVF rupture could have occurred with the patient in a prone position without the prior embolization. The patient might have presented with congestive heart failure if we had not performed prior embolization for this AVF.

\section{Conclusions}

In summary, we treated spinal intraosseous AVF by performing embolization and long posterolateral fusion with instrumentation but without anterior reconstruction. The finding of a flow void on T2-weighted MRI and preoperative embolization were important for treating the spinal intraosseous AVF. On the basis of our experience in the present case, we believe that it may not be necessary to perform vertebroplasty or anterior reconstruction for the treatment of spinal intraosseous AVF.

\section{References}

1. Iwakura T, Takehara Y, Yamashita S, Nasu H, Unno N, Nishiyama M, et al: A case of paraspinal arteriovenous fistula in the lumbar spinal body assessed with time resolved three-dimensional phase contrast MRI. J Magn Reson Imaging 36:1231-1233, 2012

2. Jin YJ, Chung SK, Kwon OK, Kim HJ: Spinal intraosseous arteriovenous fistula in the fractured vertebral body. AJNR Am J Neuroradiol 31:688-690, 2010

3. Kiyosue H, Tanoue S, Okahara M, Hori Y, Kashiwagi J, Mori H: Spinal ventral epidural arteriovenous fistulas of the lumbar spine: angioarchitecture and endovascular treatment. Neuroradiology 55:327-336, 2013

4. Papadoulas S, Konstantinou D, Kourea HP, Kritikos N, Haftouras N, Tsolakis JA: Vascular injury complicating lumbar disc surgery. A systematic review. Eur J Vasc Endovasc Surg 24:189-195, 2002

\section{Author Contributions}

Conception and design: all authors. Acquisition of data: all authors. Analysis and interpretation of data: all authors. Drafting the article: all authors. Critically revising the article: all authors. Reviewed submitted version of manuscript: all authors. Approved the final version of the manuscript on behalf of all authors: Imajo. Study supervision: all authors.

\section{Correspondence}

Yasuaki Imajo, Department of Orthopaedic Surgery, Yamaguchi University Graduate School of Medicine, 1-1 Minamikogushi, Ube, Yamaguchi, 755-8505, Japan.email: i-yasuak@ yamaguchi-u.ac.jp. 